\title{
A Two-Stage Chance Constrained Volt/Var Control Scheme for Active Distribution Networks with Nodal Power Uncertainties
}

\author{
Firdous Ul Nazir, Bikash C. Pal, Fellow, IEEE, and Rabih A. Jabr, Fellow, IEEE
}

\begin{abstract}
Volt/var control (VVC) is one of the primary functions of the distribution management system aiming at optimum operation of power distribution networks while respecting all of their operational and security constraints. However, the recent huge integration of highly stochastic distributed generation (DG) sources with the grid presents a significant challenge to the traditional VVC schemes, which assume the future to be perfectly known. This paper presents a two-stage chance constrained optimization scheme to handle these nodal power uncertainties and guarantees that the operational and security constraints are respected for almost all realizations of the uncertainty. The chance constrained model is solved by collecting enough randomly chosen samples from the probability spaces of the uncertain parameters so that the class of the problem, a mixed integer second order cone program (MISOCP), is not elevated. The algorithm not only dispatches the optimum schedule for discrete controlling devices like transformers and shunt capacitors but also optimizes the predefined decision rules for reactive power control of DG sources, thus falling in line with the requirement laid down in the revised IEEE 1547 Standard. Numerical simulations on three different test systems show the superiority of the proposed algorithm over the traditional deterministic methods.
\end{abstract}

Index Terms-Chance constrained optimization, distributed generation, radial distribution systems, volt/var control.

\section{NOMENCLATURE}

$\begin{array}{ll}\begin{array}{l}\text { I. Notation } \\ \mathbb{E}(Z)\end{array} & \text { Expected value of the random variable } Z . \\ \mathbb{P}(.) & \text { Probability of occurrence of an event. } \\ x / \bar{x} & \text { Minimum/maximum value of quantity } x . \\ \text { Cap } / D G & \text { Set of all the buses. } \\ L & \text { Set of buses with capacitors/DGs. } \\ s & \text { Set of all the branches. } \\ \operatorname{Trf} & \text { Index of } s^{\text {th }} \text { scenario. } \\ I I . P a r a m e t e r s & \text { Set of branches with transformers/OLTCs. } \\ \alpha_{P, j} / \beta_{P, j} & \text { constant power component of real/reactive } \\ & \text { power load at bus } j . \\ \alpha_{Z, j} / \beta_{Z, j} & \text { coefficient of constant impedance component } \\ & \text { of real/reactive power load at bus } j . \\ \pi & \text { Probability of occurrence of a scenario. }\end{array}$

F. Ul Nazir is with the Electrical and Electronic Engineering Department, Imperial College, London SW7 2AZ, U.K. (e-mail: f.ulnazir16@imperial.ac.uk).

B. C. Pal is with the Electrical and Electronic Engineering Department, Imperial College, London SW7 2AZ, U.K. (e-mail: b.pal@imperial.ac.uk).

R. A. Jabr is with the Deparment of Electrical \& Computer Engineering, American University of Beirut, Beirut 1107 2020, Lebanon (email:rabih.jabr@aub.edu.lb). $\sigma^{d}$

$b_{c, j}^{k}$

$N S$

$P_{j}^{d} / Q_{j}^{d}$

$r_{i j} / x_{i j}$

$S_{j}$
$t_{i j}^{k}$

$y_{j}$

$y_{i j}$

$Z_{i j}$

III. Variables

$A_{j}, B_{j}, C_{j}$

$I_{i j}$

$l_{i j}$

$P_{j} / Q_{j}$

$P_{i j} / Q_{i j}$

$p f^{d}$

$T_{c, j} / T_{o, i j}$

$u_{j}$
$w_{j}^{k} / w_{i j}^{k}$

$z_{j}^{k} / z_{i j}^{k}$

\footnotetext{
$\mathbf{V}$
}

T OLT/VAR control in distribution systems primarily aims at maintaining an acceptable voltage profile throughout the system by strictly restricting the voltage magnitude at each node within its upper and lower bounds. There can also be 
secondary objectives such as active power loss minimization, and power demand minimization on the substation which is also called energy conservation [1]. VVC achieves this aim by determining a periodical schedule of switched shunt capacitores, load tap changing transformers, and reactive power from DG sources.

The majority of the VVC strategies in distribution systems employ a centralized controller which decides the optimum control set points of various controlling devices based on current network topology and net nodal injections. These methods use a mathematical representation of the system together with real-time measurements and employ optimization techniques to guarantee an optimum solution [2],[3]. The common approaches used to solve the centralized VVC problem include the well-known interior point methods [4], mixed integer linear programming [5], dynamic programming [6],[7], and evolutionary algorithms such as genetic algorithms [8] and particle swarm optimization [9].

The power industry is undergoing a phenomenal transformation to achieve low carbon targets [10], by increasing the share of renewables in its energy mix. The huge integration of these renewable based DG sources creates many operational challenges for the traditional voltage control strategies. Wind and solar power are highly intermittent in nature besides being non-dispatchable [11], whereas the traditional synchronous generator output is free from any uncertainties, so is taken to be deterministic for power system operation and control applications. The problem is compounded by the fact that distribution systems are characterized by a high resistance to reactance ratio, which makes voltage variations more susceptible to fluctuations in the nodal real power injections. In this regard, several researchers have recently focussed on modelling the VVC problem in a stochastic framework. The authors in [12] considered the uncertainties present in hourly solar irradiance, wind power and load forecasts to set up a stochastic multiobjective VVC problem which was solved by using an evolutionary technique known as the modified teaching learning algorithm. In [13], the stochasticity present in the forecasts was dealt by setting up a robust reactive power optimization framework which was subsequently solved by an approach similar to Bender's decomposition, known as the column and constraint generation algorithm. A chance constrained approach to solve the stochastic VVC problem was considered in [14], whose solution algorithm was more or less similar to the gradient descent algorithm. However, the solutions required a posteriori validation through Monte Carlo trials because of the non-convex nature of the power flow equations.

This paper builds on the stochastic VVC problem formulation through a chance constrained framework, resulting in the security constraints being met for almost all the realizations of uncertainty with a certain confidence level. The uncertainties in the system, including the DG and load forecast errors, are modelled using a scenario based stochastic approach so that the class of the problem is not elevated. A scenario reduction algorithm based on submodular optimization [15] is used to choose a subset of scenarios which contains most of the stochastic information present in the original set. The discrete nature of transformer tap settings and shunt capacitors together with the nonlinear power flow equations results in a mixed integer nonlinear programming (MINLP) problem which is known to be NP hard and cannot be reliably solved due to the non-convex nature of the solution space. Thus a conic relaxation technique based on the branch flow model [16] is used to relax the original non-convex power flow equations, which transforms the MINLP model to a mixed integer second order cone program (MISOCP); MISOCP can be solved using state-of-the-art commercial solvers. The developed model is also able to provide a recourse opportunity to the decision maker by incorporating quadratic decision rules which decide real time inverter reactive power as a function of DG active power, a functionality that is completely alien to the traditional VVC schemes [1]-[9]. The DGs were prohibited to lend any reactive power support to the system by the IEEE 1547 standard [17] until recently, when an amendment [18] to the standard allowed DG reactive power to actively participate in distribution voltage regulation. This gives rise to a twostage stochastic VVC framework where the classical discrete controlling devices are scheduled in the first stage and held at fixed settings throughout the entire optimization horizon, while the inverter reactive powers are adjusted in the second stage upon the final revelation of uncertainty. This is justified because of concerns about wear and tear and the inherent slow controlling speed of discrete controlling devices as compared to the DG inverters. The developed VVC framework allows the DG owners to run their DGs at the possible maximum power point without any active power curtailment issues, which ensures that the share of clean renewable energy is maximized in the energy mix of the network. The only requirement by the VVC is that the DG owners should be able to provide an estimate of the next optimization horizon forecast of active power with the corresponding confidence measurements like the standard deviation of the assumed probability distribution function. Thus, the paper attempts to solve a chance constrained optimization problem which has traditionally been solved by reformulation of the non-convex probabilistic constraints to their deterministic counterparts using the probability density functions [19]- [20]. As it turns out that the deterministic counterparts are more complex than the original basic constraints, free of probabilities, like a linear chance constraint results in a second order conic constraint. Thus the original chance constrained version is said to have been elevated in complexity to the more complex deterministic optimization problem. Also carrying out this reformulation is very difficult in practice. This is particularly true because of the fact that the power flow equations involve trigonometric quantities when presented in their polar form. However the scenario approach used in this research preserves the basic structure of the bounds, used as chance constraints. This means the class of the basic MISOCP problem is not elevated. Further, we anticipate that the proposed algorithm to solve the scenario based chance constrained VVC can be applied to other chance constrained optimal power flow problems like unit commitment, economic dispatch etc. without much modifications. The proposed algorithm in its current form runs in a centralized fashion like standard VVC applications; 
it requires communication and monitoring infrastructure to function properly. The handling of uncertainty also limits how the algorithm scales to very large systems. However the research will serve as a starting point to develop VVC routines that handle uncertainty, and which could be extendible to decentralized applications.

The rest of the paper is organised as follows: Section II introduces the general chance constrained VVC problem framework in active distribution networks and presents ways to handle the challenges that are related to the non-convex nature of the power flow solution space and the computational complexity of probabilistic security constraints. Section III presents a two-stage stochastic formulation of the VVC problem taking into account the nodal net injection uncertainties; a quadratic rule for adjusting DG reactive power as a function of its real power is also presented. A step by step solution algorithm is proposed to solve the two-stage stochastic optimization problem in Section IV. Section V reports numerical results on three different test systems and a comparison with the classical VVC schemes; the comparison demonstrates the superiority of the proposed approach. The paper is concluded in Section VI.

\section{Chance Constrained Volt/Var Control PROBLEM FOR DISTRIBUTION NETWORKS}

The general chance constrained VVC problem uses probabilistic security constraints and aims to minimize the meanvalue of the power loss in the system. This problem in a distribution system is modelled in the following manner:

$$
\begin{array}{rl}
\min _{u} & \mathbb{E}\left(\mathrm{P}_{l}\right) \\
\text { s.t. } & f(y, u, \zeta)=0 \\
& \mathbb{P}\left(\left|V_{i}\right| \leq\left|V_{i}\right| \leq \overline{\left|V_{i}\right|}\right) \geq 1-\epsilon, \\
& \mathbb{P}\left(\left|I_{i j}\right| \leq \overline{\left|I_{i j}\right|}\right) \geq 1-\epsilon, \\
& \underline{p f^{d}} \leq p f^{d}
\end{array}
$$$$
\forall i \in B
$$$$
\forall(i, j) \in L
$$

The equality constraint in the above framework denotes the power flow equations, which must be satisfied for every solution instance of the problem. This formulation takes into account the randomness of the uncertain parameter $\zeta$ and forces the power system security constraints up to a desired probability level $(1-\epsilon)$, thus the solutions are said to be $\epsilon$ level robustly feasible. This formulation faces two important challenges: i) non-convex nature of the power flow equations, ii) computation of probability spaces for bus voltages and branch currents.

\section{A. Convexification of power flow equations}

The distribution system is a radial network and hence can be modelled by a set of recursive equations, called the branch flow equations [16]. The branch flow model is built by following three equations:

$$
\begin{aligned}
& V_{i}-V_{j}=Z_{i j} I_{i j} \\
& S_{i j}=V_{i} I_{i j}^{*} \\
& S_{j}=\sum_{k: j \rightarrow k} S_{j k}-\sum_{i: i \rightarrow j}\left(S_{i j}-Z_{i j}\left|I_{i j}\right|^{2}\right)+y_{j}^{*}\left|V_{j}\right|^{2}
\end{aligned}
$$

(2) represents Ohm's law while (3) denotes the complex power flowing over a branch from node $i$ to node $j$. The branch power flow model is completed by (4), which denotes the complex power balance at each node. This set of equations is transformed to a real-variable formulation which is more easily handled by optimization algorithms. Separating the real and reactive parts of complex quantities and including transformer tap ratios and switched capacitors gives:

$$
\begin{aligned}
& P_{j}+P_{j}^{d}=\sum_{k: j \rightarrow k} P_{j k}-\sum_{i: i \rightarrow j}\left(P_{i j}-r_{i j} l_{i j}\right)+g_{j} u_{j} \\
& Q_{j}+u_{j} b_{c j}+Q_{j}^{d}+b_{j} u_{j}=\sum_{k: j \rightarrow k} Q_{j k}-\sum_{i: i \rightarrow j}\left(Q_{i j}-x_{i j} l_{i j}\right) \\
& \frac{u_{j}}{t_{i j}^{2}}=u_{i}-2\left(r_{i j} P_{i j}+x_{i j} Q_{i j}\right)+\left(r_{i j}^{2}+x_{i j}^{2}\right) l_{i j} \\
& P_{i j}^{2}+Q_{i j}^{2}=l_{i j} u_{i}
\end{aligned}
$$

The shunt admittance $y_{j}=g_{j}+i b_{j}$. The equations (5a) - (5c) are linear in the variables $P_{j}, P_{i j}, Q_{j}, Q_{i j}, u_{i}, l_{i j}, b_{c j}$ and $t_{i j}^{2}$ with the exception of the bilinear terms $u_{j} b_{c j}$ and $\frac{u_{j}}{t_{i j}^{2}}$ in (5b) and (5c) respectively. If there is no capacitor connected at a particular bus, then the term $u_{j} b_{c j}$ will not appear in the equation pertaining to that bus and if there is no step voltage regulator present on the branch $(i, j)$, then the corresponding $t_{i j}$ is set at unity. Besides bilinear terms, (5d) gives rise to a non-convex region. These issues can be simplified in the following way:

1) Big-M method for bilinear terms: Both the bilinear terms represent the product of a discrete variable and a continuous variable. Let us suppose that $d$ is a discrete variable which can take values in the discrete set $\left[d_{1}, d_{2}, \ldots, d_{n}\right]$ and that $c$ is a continuous variable. Also assume that there is a binary variable $z_{j}$ corresponding to each discrete level $d_{j}$ of the discrete variable. Then we can write [21]:

$$
\begin{gathered}
d c=\sum_{j=1}^{n} z_{j} d_{j} c \\
\sum_{j=1}^{n} z_{j}=1
\end{gathered}
$$

(7) is a special ordered set of type 1 and hence gives the mixed integer solver a more intelligent way of finding the optimal solution. It also signifies a practicality constraint of selecting one and only one possible level for the discrete quantity. (6) can be linearised by letting the product of $z_{j} c$ as a new variable say $w_{j}$ and adding the following linear inequality constraints:

$$
\begin{aligned}
c-M\left(1-z_{j}\right) & \leq w_{j} \leq c+M\left(1-z_{j}\right) \\
-M z_{j} & \leq w_{j} \leq M z_{j}
\end{aligned}
$$

$M$ is a big number. If $z_{j}$ acquires a value of 1 , then $w_{j}$ is forced to take the value of $c$ by (8) while (9) remains redundant. However, if $z_{j}=0$ (8) becomes an inactive constraint while (9) forces the value of $w_{j}$ to be 0.

The number of binary variables required by the big-M method is significantly reduced if the discrete set $\left[d_{1}, d_{2}, \ldots\right.$, $d_{n}$ ] is equivalent to a truncated arithmetic series with a constant difference, say $\delta$, between any two consecutive elements. 
In this case, (6) and (7) are replaced by the following highly efficient modelling [13]:

$$
\begin{array}{r}
d c=d_{1} c+\delta \sum_{k=0}^{m} 2^{k} x_{k} c \\
d_{1}+\delta \sum_{k=0}^{m} 2^{k} x_{k} \leq d_{n}
\end{array}
$$

$x_{k}$ are binary variables. Clearly the number of binary variables required by this model is much lower than the actual number of discrete entries in the support set of variable $d$. (10) can be linearised in the same way as done for (6) by introducing dummy variables and adding linear inequality constraints on these dummy variables similar to (8) and (9).

2) Conic relaxation of the quadratic equality: The quadratic equality in (5d) can be relaxed to an inequality constraint and the authors in [16] have proved that this relaxation is exact under standard and realistic assumptions in distribution network operation. The feasible set of the relaxed inequality is convex as it can be easily transformed to a second order conic constraint. The relaxation and transformation is shown below:

$$
\begin{aligned}
P_{i j}^{2}+Q_{i j}^{2}=l_{i j} u_{i} & \Longrightarrow P_{i j}^{2}+Q_{i j}^{2} \leq l_{i j} u_{i} \\
& \Longrightarrow\left\|\begin{array}{c}
2 P_{i j} \\
2 Q_{i j} \\
l_{i j}-u_{i}
\end{array}\right\|_{2} \leq l_{i j}+u_{i}
\end{aligned}
$$

\section{B. Computationally feasible approach for probabilistic con-} straints

The computation of the exact probability spaces for the bus voltages and branch currents required in framework (1) is difficult and computationally challenging. However, Esfahani et al [22] proved that if a certain minimum number $N$ of randomly chosen samples of the uncertain parameter (sampled with respect to its probability space) are collected and the constraints of the chance constrained problem are forced to be feasible for all these sampled instances, then the resulting solution (which we will call the randomized solution) will be feasible to the original chance constrained problem with a predefined confidence level. To guarantee these a priori probabilistic bounds, the deterministic optimization problem should be either convex or a mixed integer program whose continuous relaxation is convex; such a structure is referred to as a mixed integer 'convex' program (MICP). For a particular subset of MICPs whose integer variables admit values in the set $\{0,1\}$, the number of samples to be generated with $n$ continuous and $l$ binary decision variables is governed by the following relation:

$$
2^{l} \sum_{i=0}^{n-1}\left(\begin{array}{c}
N \\
i
\end{array}\right) \epsilon^{i}(1-\epsilon)^{N-i} \leq \gamma
$$

where $\gamma$ is the confidence parameter implying that with probability no smaller than $1-\gamma$, the randomized solution of the original chance constrained problem will be $\epsilon$-level robustly feasible. (14) allows to adjust $\epsilon$ according to the acceptable level of risk and $\gamma$, which will be normally small enough to be negligible. It is advocated that the value of $N$ should be chosen as large as possible; practically $N$ is selected within the computational power of the solver used.

This scenario approach of solving a chance constrained framework not only preserves the basic structure of the problem but also works equally well with any probability distribution, even when the underlying probability distribution is not known but the samples are available from measurements as is the case in most practical situations.

\section{Penalization of the tap changes for discrete controlling devices}

The concern about the wear and tear and ultimately the life of the classical voltage regulating devices requires that frequent switching operations of these devices be prevented. The minimization of tap changes generally appears as an objective term in the multi-time step optimization programs [4], like the one which schedules the voltage controlling devices for each hour in the next day, so that the total tapchanger operations are minimized over the entire scheduling horizon. However, the VVC considered in this research is a single time step optimization carried for the next hour for good reason of having reasonably accurate forecasts for the uncertain nodal injections, as against the multi-step optimization which requires day-ahead forecasts for the uncertain quantities and hence jeopardizes the robustness property of the algorithm. Hence, we consider the absolute value function of the tap changes between consecutive schedules as an objective term which resists the tap changes corresponding to the previous schedule and thus achieves the tap minimization. Thus the actual objective of the optimization program considered in framework (1) will be:

$$
\min _{u} C_{p} \mathbb{E}\left(\mathrm{P}_{l}\right)+C_{s} \mid \text { Tap }_{\text {new }}-\text { Tap old } \mid
$$

Here the weights associated with the power loss and the tap changes are denoted by $C_{p}$ and $C_{s}$ respectively. We consider the weights such that $C_{p}+C_{s}=1$, which implies that the decision maker can run the optimization routine with a preferential setting for either of the two objective terms. If $C_{p}=1$, only power loss minimization is considered while a value of $C_{p}=0$ means only tap minimization is considered. In order to run the VVC in an unbiased manner, both $C_{p}$ and $C_{s}$ are set equal to 0.5 . The absolute value function appearing in the objective can be pushed in the constraint set of the program by using the epigraph programming concept [23]:

$$
\begin{aligned}
\min _{u} & C_{p} \mathbb{E}\left(\mathrm{P}_{l}\right)+C_{s} T \\
\text { s.t. } & -T \leq\left(\text { Tap }_{\text {new }}-\text { Tap }_{\text {old }}\right) \leq T
\end{aligned}
$$

Here, $T \geq 0$ is the upper bound on the number of tap changes in the current schedule relative to the previous schedule.

\section{Two Stage Stochastic Optimization Formulation fOR Volt/VAR CONTROL PROBlem}

After incorporating the simplifications discussed in Section II in the framework (1), a two-stage stochastic VVC problem 
is set up: the slow controls viz. capacitor, OLTC and step voltage regulator set points are "here and now" decisions that need to be made before the uncertainty is revealed and are kept fixed throughout the entire optimization interval - an hourly time window, whereas the fast controls viz. inverter reactive power is allowed to be a second stage recourse or "wait and see" decision. The second stage adjusts the reactive power from the DGs in real time according to a predefined rule. This pre-defined rule is chosen to be a univariate quadratic equation relating the DG reactive power to its active power, whose coefficients are also available from the first stage. Although the developed framework is flexible enough to optimize over a wide range of decision rules, the quadratic rule was chosen because it closely conforms with the standard $\mathrm{Q}(\mathrm{P})$ characteristic piecewise linear curves as suggested in the German grid code [24]. However if the $\mathrm{Q}(\mathrm{P})$ characteristics of a DG are not adequately approximated by a quadratic rule like the case of a saturation function or a saturation function with a dead zone, different decision rules need to be adopted or else the corresponding DG will not be able to participate in reactive power ancillary services. It is important to note here that the algorithm allows the use of any polynomial reactive power rule, in which case the corresponding constraint is always linear. Previously a linear rule to adjust the DG reactive power has been used in the literature [25], however the benefit of a quadratic reactive power rule is that the quadratic term provides additional recourse flexibility over the linear rule. Also the optimizer can force the coefficient of the quadratic term to be zero if a linear rule is necessary. The pre-defined rule adopted in this study has the following general form:

$$
Q_{j}^{d}=A_{j}+B_{j} P_{j}^{d}+C_{j}\left(P_{j}^{d}\right)^{2}
$$

Thus, in the first stage the following MISOCP is solved:

$$
\begin{aligned}
& \min _{O} C_{p} \sum_{s=1}^{N S} \pi^{s} \sum_{(i, j) \in L} r_{i j} l_{i j}^{s}+C_{s}\left(\sum_{j \in C a p} T_{c, j}+\sum_{(i, j) \in T r f} T_{o, i j}\right. \\
& \text { s.t. }-T_{c, j} \leq \underline{b_{c, j}}+\delta_{j} \sum_{k=0}^{m_{j}} 2^{k} z_{j}^{k}-b_{c, j, o l d} \leq T_{c, j}, \quad \forall j \in \text { Cap } \\
& -T_{o, i j} \leq \sum_{k=1}^{n_{j}} z_{i j}^{k} t_{i j}^{k}-t_{i j, o l d} \leq T_{o, i j}, \quad \forall(i, j) \in \operatorname{Trf} \\
& \underbrace{\alpha_{P, j}^{s}+\alpha_{Z, j}^{s} u_{j}^{s}}_{P_{j}^{s}}+P_{j}^{d, s}=\sum_{k: j \rightarrow k} P_{j k}^{s}-\sum_{i: i \rightarrow j}\left(P_{i j}^{s}-r_{i j} l_{i j}^{s}\right) \\
& +g_{j} u_{j}^{s}, \quad \forall j \in B, \forall s=1, \ldots, N S \\
& \underbrace{\beta_{P, j}^{s}+\beta_{Z, j}^{s} u_{j}^{s}}_{Q_{j}^{s}}+\underbrace{b_{c, j}^{m i n} u_{j}^{s}+\delta_{j} \sum_{k=0}^{m_{j}} 2^{k} w_{j}^{k, s}}_{b_{c, j} u_{j}^{s}} \\
& +\underbrace{A_{j}+B_{j} P_{j}^{d, s}+C_{j}\left(P_{j}^{d, s}\right)^{2}}_{Q_{j}^{d, s}}+b_{j} u_{j}^{s}=\sum_{k: j \rightarrow k} Q_{j k}^{s}
\end{aligned}
$$

The objective of the above optimization problem as defined by (19) is to minimize the expected power loss in the network across all the scenarios while keeping the schedule of the discrete controlling devices close to the previous schedule. The feasible region for this problem is demarcated by the constraints (20)-(39) and is defined by the vector of optimization variables, $O=\left[z_{j}^{k}, z_{i j}^{k}, T_{c, j}, T_{o, i j}, w_{j}^{k, s}, w_{i j}^{k, s}, A_{j}, B_{j}, C_{j}, P_{i j}^{s}\right.$, 
$\left.Q_{i j}^{s}, l_{i j}^{s}, u_{j}^{s}\right]^{T}$. (20) and (21) make sure that the current schedule is not far from the previous schedule through the concept of epigraph programming as is discussed in (17). (22) describes the real power balance at each node of the system. Note that the ZP model is used for the net load real power injection at a node with $\alpha_{P, j}$ and $\alpha_{Z, j}$, which define the constant power and the constant impedance component coefficients of the load injection $P_{j}$. The full ZIP load modelling is not directly amenable with the second order cone relaxations, however an approximate ZIP modelling is possible on the lines of [26]. Eq. (23) describes the reactive power balance at each node of the system. The bilinear term $b_{c, j} u_{j}$ is linearised by the efficient procedure as explained in (10)-(11) through the introduction of dummy variables $w_{j}^{k}$. The number of binary variables $z_{j}^{k}$ required by this efficient modelling is bounded by (24). The constraints for these dummy variables are defined by (25) and (26) in the same vein as (8) and (9). (28) and (29) define the Ohm's law equation of the branch flow model. Eq. (28) is defined for a branch which does not feature a transformer, while (29) is necessary for the remaining network branches. Eq. (29) is basically the same as (5c) with the bilinear term $\frac{u_{j}}{t_{i j}^{2}}$ linearised in accordance with (6). This linearisation is forced to be equivalent to the original constraint with the help of the Big-M method defined through (30)-(33). (34) gives the second order cone relaxation as described by (13). Eq. (35) defines the voltage security constraints of the network while the branch current security constraints are defined by (36) and (37). These branch current security constraints correspond to the currents starting at the ends nodes of the branches and take into account the shunt current components flowing through the transversal elements of the equivalent- $\pi$ model. There is no way for the program to confirm, in advance or during optimization, which branch will exhibit reverse power flow; therefore the current magnitude squared from each end of the branch is used. In case of normal power flow (36) will be active while as (37) will be redundant and for reverse power flows (37) will be active with (36) being redundant. The amount of reactive power available from each DG is limited by (38), which defines a power factor type control that implies the DG power factor can not be deteriorated beyond $\left|p f^{d}\right|$.

\section{Proposed Solution Strategy}

The two-stage stochastic VVC problem is set up to ensure that the voltage violation probability, during the optimization horizon, is kept under desirable limits in the face of uncertain nodal net injections. The algorithm requires to simultaneously consider all the generated scenarios leading to a huge optimization program. In order to keep the program tractable, only a small but appropriate subset of the generated scenarios need to be considered. To this end an algorithm is proposed which is summarized by the following steps:

1: Load the power system line data, predicted value of load and DG data. Initialize the iteration counter to 1 . Initialize the solution-scenario-set by the predicted value of load and DG data. The solution-scenario-set contains all the scenarios that the optimization program has to consider in the current iteration. Thus in the first iteration, the scenario based chance constrained algorithm is equivalent to the base case VVC algorithm.

2: Solve the base case VVC problem by considering only the predicted values of the uncertain quantities. The base case VVC problem determines the schedule of voltage controlling devices considering the forecast values of the nodal injections free of any error and hence deterministic in nature. The base case problem is only a special case of the model presented by the Eqs. (19)-(39), which can be obtained by considering only one scenario of predicted values of load and DG data. In other words, the superscript $s$ will not appear on any quantity in these equations when treating it as a base case VVC problem. Compute the schedule of the slow controlling devices viz. OLTC, VR and capacitors. Also store the value of the quadratic rule coefficients relating DG active and reactive power. Since this schedule of the controlling devices corresponds to the base case VVC, it needs to be checked for its robustness level.

3: From the probability distribution functions of the forecast errors, generate $N$ number of forecast-error-scenarios as dictated by (14). Compute $N$ different realizations (called scenarios from now onwards) of uncertain data by adding forecast-error-scenarios with predicted values of the load and DG data [27].

4: Form the bus admittance matrix, $Y_{b u s}$, that accounts for the most recent schedule of slow controlling devices. The most recent schedule in the first iteration corresponds to the schedule computed in step 2. Carry out a proper ordering of the columns of $Y_{\text {bus }}$ for factorization sparsity and perform its LU factorization; 'LU' stands for 'lower upper'. This permutation of the columns, which is based on the sparsity pattern of $Y_{\text {bus }}$, ensures that the fill ins in the LU factors are reduced.

5: Compute $N$ different power flow solutions using the current injection load flow algorithm [28] for the $N$ different scenarios generated in step 3 . Note that the $Y_{\text {bus }}$ remains constant for all these load flows, which implies the LU factors computed in the previous step are constant for all the $N$ power flows. This ensures that the load flow solutions for all the generated scenarios are available quickly enough for the whole algorithm to converge within practical times. The buses hosting DGs are allowed to inject reactive power according to the most recent coefficients of the quadratic decision rule, which relates the DG real and reactive power. Check the load flow solution of each scenario for any security constraint violation and hence collect the set of violated scenarios. Let the cardinality of the violated scenario set be $N_{V}$.

6: If $N_{V}=0$, stop and dispatch the schedule of classical slow controlling devices and the coefficients of the quadratic decision rule, else go to step 7. Note that when $N_{V}$ becomes zero, the current schedule is sufficient to drive the security constraints of all the scenarios to their feasible region. A non-zero value of $N_{V}$ implies that there are still some scenarios whose security constraints are infeasible based on the current schedule.

7: Choose the most dominant subset of cardinality $N_{V R}$ 
TABLE I

MINIMUM $N$ FOR DIFFERENT TEST CASES FOR $\gamma=1 e-10$ AND $\epsilon=0.02$

\begin{tabular}{|c|c|c|c|c|c|c|c|c|}
\hline \multicolumn{3}{|c|}{95 bus system } & \multicolumn{3}{c|}{123 bus system } & \multicolumn{3}{c|}{190 bus system } \\
\hline$n$ & $l$ & $N$ & $n$ & $l$ & $N$ & $n$ & $l$ & $N$ \\
\hline 499 & 107 & 16272 & 573 & 48 & 13722 & 997 & 214 & 19955 \\
\hline
\end{tabular}

from the violated scenario set by running submodular scenario reduction algorithm [29]. This algorithm is basically an accelerated greedy algorithm which seeks to maximise a particular submodular function in the $l_{2}$-norm of the original and the reduced set of scenarios. The scenario reduction algorithm not only effectively trims down the size of violated scenario set but also retains most of the stochastic information embedded in the set. The probabilities of the original scenario set are redistributed according to the rule proposed in [30], according to which the probability of a preserved scenario is given by the sum of its own probability and the probability of all the deleted scenarios which are closest to it with respect to their Euclidean distances. Add these $N_{V R}$ scenarios to the solution-scenario-set.

8: Run the stochastic scenario based chance constrained VVC optimization algorithm as set up in Section III taking into account the solution-scenario-set only. Update the schedules of slow controlling devices and coefficients of the quadratic decision rule.

9: Increment the iteration counter by 1 and go to step 4 .

It can be noticed that the MISOCP set up in (19) - (39) is basically solved in the step 8 of the above algorithm. The preceding steps of the algorithm are required to prepare the solution-scenario-set which comprises of a set a violated scenarios, that are able to represent the majority of the stochastic information about the set of violated scenarios while at the same time being considerably smaller in size. The algorithm behaves in such a way so as to guarantee that the schedule obtained in the final iteration is robust enough to respect the security constraints of all the scenarios generated in the step 3 of the algorithm, by considering only handful of them. This algorithm is easy to implement and ensures to remove almost all the voltage violations in the face of uncertainty. The algorithm also provides intuitive guidelines on how the powerful technique of scenario based optimization can be used to obtain robust solutions for various other power system operation and control applications like OPF, unit commitment, economic dispatch etc. The corresponding flowchart is shown in Fig. 1.

\section{NumericAl RESUlts}

\section{A. System Model}

The computational tasks were performed on a $3.5 \mathrm{GHz}$ Intel Xeon E5 processor with 64 GB of RAM, where the proposed algorithm was programmed in MATLAB and the MISOCP problem was solved using the CPLEX 12.7 optimization studio. The algorithm was tested on the following systems:

- The UKGDS 95 bus system [31] which is fed by a 33/11 $\mathrm{kV}$ substation OLTC transformer is shown in Fig. 2. A

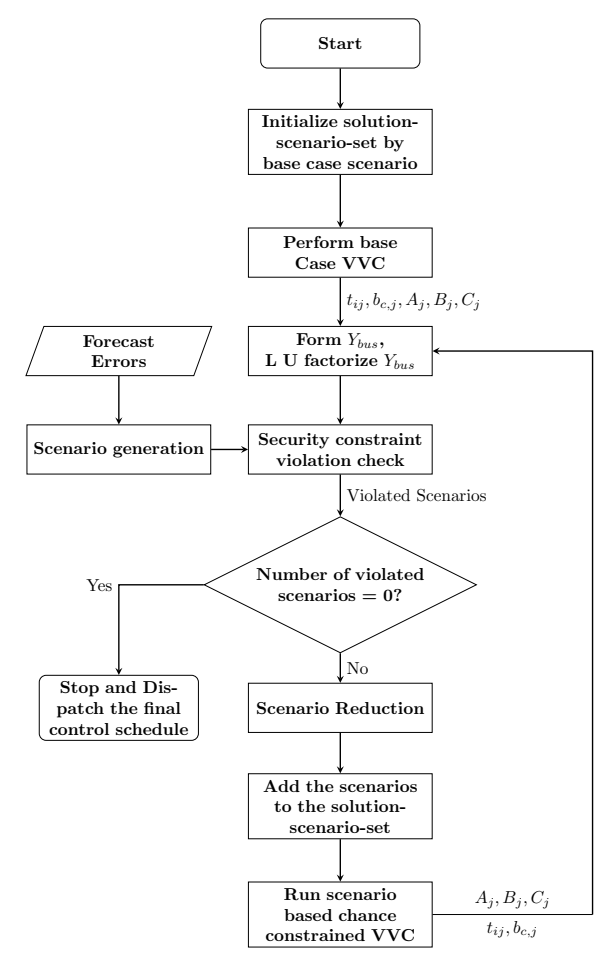

Fig. 1. Solution algorithm flowchart

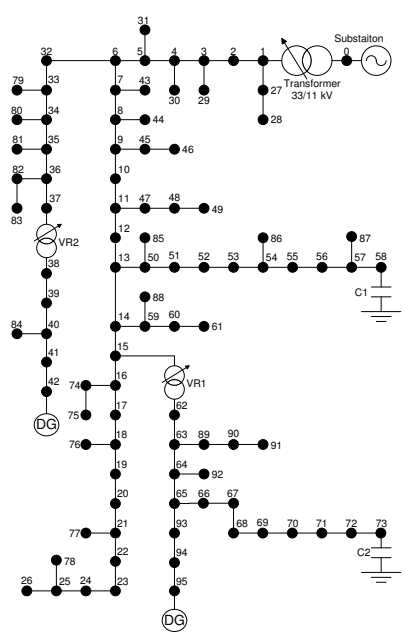

Fig. 2. UKGDS 95 bus system

base voltage of $11 \mathrm{kV}$ and a base power of 10 MVA are assumed. There are two step voltage regulators (VR) VR1 between buses 15-62 and VR2 between buses 3738. The OLTC and the VRs have an operating range of 0.9 to 1.1 spanned by 33 equal steps. There are also two switchable shunt capacitors (SSC) - SSC1 hosted at bus 58 and SSC2 at bus 73. Both the SSCs can supply a maximum of 300 kvar with each having a step size of 20 kvar. Two DG sources at buses 42 and 95 are considered. The forecasted DG power at bus 42 is $1.1 \mathrm{MW}$ while that at bus 95 is $1.2 \mathrm{MW}$.

- The positive sequence IEEE 123 bus network, shown in 


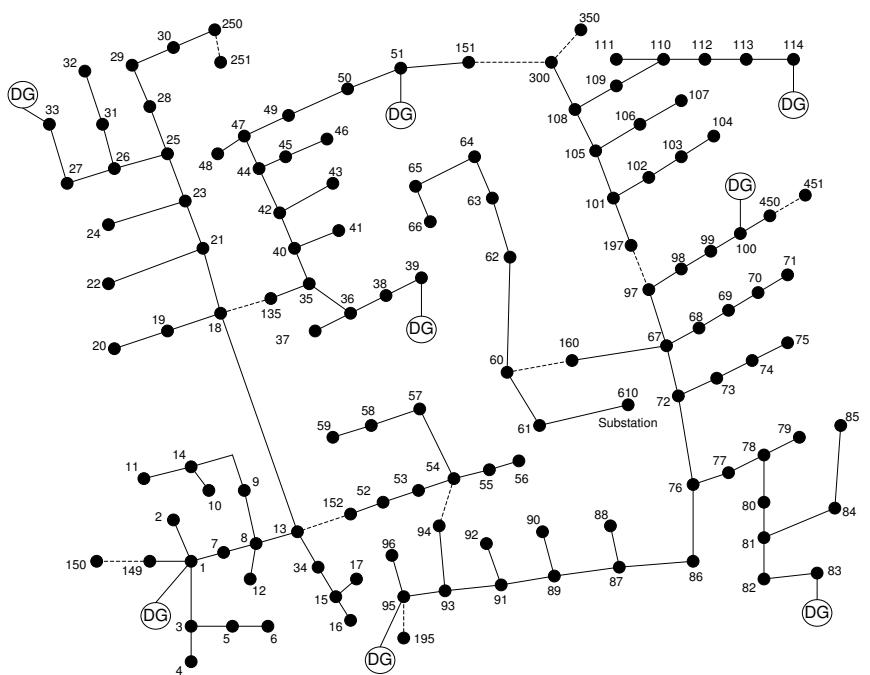

Fig. 3. IEEE 123 bus system

TABLE II

Control Set Points of Classical Reactive Power Control DEVICES FOR THE 95 BUS UKGDS SYSTEM

\begin{tabular}{|c|c|c|c|c|c|c|}
\hline & $\sigma^{d}$ & $\begin{array}{c}\text { SSC-1 } \\
\text { kvar }\end{array}$ & $\begin{array}{c}\text { SSC-2 } \\
\text { kvar }\end{array}$ & TR & VR-1 & VR-2 \\
\hline Det & - & 60 & 60 & 1.04375 & 0.98125 & 1.0000 \\
\hline \multirow{4}{*}{ CC } & 0.10 & 60 & 60 & 1.03125 & 0.98125 & 1.00625 \\
\cline { 2 - 7 } & 0.15 & 80 & 60 & 1.01875 & 0.97500 & 1.01250 \\
\cline { 2 - 7 } & 0.20 & 60 & 60 & 1.02500 & 0.96875 & 1.00000 \\
\cline { 2 - 7 } & 0.25 & 60 & 60 & 1.03125 & 0.98750 & 0.98750 \\
\hline
\end{tabular}

Fig. 3, is used for further tests. There are 8 DGs (at the nodes $114,51,95,100,1,39,33$, and 83), 5 SSCs ( at the buses 54, 108, 35, 72, and 12) and 3 OLTCs (on the branches 610-61, 160-67, and 25-26) in this network. All the DGs have a forecast real power injection of $0.5 \mathrm{MW}$. Every SSC allows eight discrete steps with constant step length of $50 \mathrm{kvar}$ and hence can supply a maximum of 350 kvar. The OLTCs operate with a tap step size of 0.01 and the range is [0.95-1.05].

- Another set of tests is performed on a 190 bus network which is created by appending another UKGDS 95 bus system to the one shown in Fig. 2, at node 95. The numbering of the nodes on the appended portion is in the same sequence as the parent network. The loads in the appended networks are slightly modified from the original. There are six OLTCs, of same specification as the parent network, on the branches $0-1,15-62,37-38$, 95-96, 130-131, and 158-59. The network also hosts four SSCs of similar specifications as the UKGDS 95 bus system at the nodes $58,73,137$, and 186 . The forecast power of each of the four DGs at the buses 42, 95, 121, and 168 is $0.5 \mathrm{MW}$.

The capacity of the interfacing DG inverters in each case is overrated so that they can operate at 0.95 lead/lag pf during worst conditions. The forecast errors of the loads and DG power are assumed to be Gaussian [14], with the standard deviation of the load forecast errors set equal to $5 \%$ of the nominal value [32].
TABLE III

CoEfFicients of QuadRatic Rule BetweEn DG ACTIVE AND REACTIVE POWERS FOR THE 95 BUS UKGDS SYSTEM

\begin{tabular}{|c|c|c|c|c|c|c|c|}
\hline \multirow{2}{*}{} & $\sigma^{d}$ & \multicolumn{3}{|c|}{ DG at Bus at 42 } & \multicolumn{3}{c|}{ DG at Bus 95 } \\
\cline { 3 - 8 } & & $\mathrm{A}$ & $\mathrm{B}$ & $\mathrm{C}$ & $\mathrm{A}$ & $\mathrm{B}$ & $\mathrm{C}$ \\
\hline \multirow{2}{*}{ Det } & - & 0.0362 & 0 & 0 & 0.0092 & 0 & 0 \\
\hline \multirow{4}{*}{$\mathrm{CC}$} & 0.10 & -0.0207 & 0.7830 & -2.4847 & -0.0426 & 0.9553 & -4.2604 \\
\cline { 2 - 8 } & 0.15 & -0.0033 & 0.4537 & -1.0692 & -0.0053 & 0.3055 & -1.2849 \\
\cline { 2 - 8 } & 0.20 & -0.0017 & 0.4176 & -1.0874 & -0.0223 & 0.5956 & -2.4770 \\
\cline { 2 - 8 } & 0.25 & -0.0013 & 0.4785 & -1.2543 & -0.0005 & 0.2327 & -1.2480 \\
\hline
\end{tabular}

TABLE IV

SECURITY CONSTRAINT VIOLATIONS COMPARISON FOR THE 95 BUS UKGDS SYSTEM

\begin{tabular}{|c|c|c|c|c|c|}
\hline \multirow{2}{*}{$\sigma^{d}$} & \multicolumn{2}{|c|}{ SFR\% } & \multicolumn{2}{c|}{ VVP\% } & \multirow{2}{*}{ CI\% } \\
\cline { 2 - 5 } & Det & CC & Det & CC & \\
\hline 0.10 & 42.18 & 0.67 & 6.50 & 0.01 & 2.29 \\
\hline 0.15 & 48.86 & 0.28 & 10.92 & 0 & 5.86 \\
\hline 0.20 & 53.22 & 0.11 & 14.75 & 0 & 6.34 \\
\hline 0.25 & 55.85 & 5.67 & 17.91 & 0.25 & 3.18 \\
\hline
\end{tabular}

In order to estimate the sample size $N$ from (14), the value of $\epsilon$ is set at 0.02 implying a $98 \%$ robustness level of the optimizer while the confidence parameter $\gamma$ is chosen to be $1 e-10$. In order to guarantee these thresholds, the minimum sample size necessary for the three cases under study is shown in Table I. The number of scenarios considered in this study is 30000 for each of the three cases, which is sufficient to guarantee that almost with full confidence, the randomized solution obtained is $98 \%$ robustly feasible for the original chance constrained program.

The test results discussed below in subsections $B$ and $C$ have been carried out considering only the power loss minimization as the objective of the VVC routine, i.e. assuming $C_{p}=1$. However, the last subsection takes into account both the power loss and tap minimization as the objective.

\section{B. Comparison of Solutions Between Two Stage Stochastic and Deterministic VVC}

The schedules obtained from base case and stochastic twostage VVC (STS-VVC) case are validated against 50,000 Monte-Carlo (MC) trials in each different environment. The effectiveness of the STS-VVC over the base case algorithm is determined by parameters such as the scenario failure rate (SFR) and voltage violation probability (VVP). These parameters are defined as follows:

SFR: SFR is defined as the fraction of scenarios which have at least one voltage violation.

VVP: VVP is defined as the fraction of the total node voltage violations across all the MC trials. Mathematically it can be defined as:

$$
V V P=\frac{\sum_{i=1}^{n M C} n V V_{i}}{n M C * n B}
$$

where $n M C$ denotes total number of Monte-Carlo trials, $n V V_{i}$ denotes the number of voltage violations in $i^{t h}$ trial, and $n B$ is the number of buses in the system.

The level of conservativeness of the STS-VVC solutions against those of the base case is quantified by introducing a 
TABLE V

SECURITy CONSTRAINT VIOLATIONS COMPARISON FOR THE IEEE 123 BUS SYSTEM

\begin{tabular}{|c|c|c|c|c|c|}
\hline \multirow{2}{*}{$\sigma^{d}$} & \multicolumn{2}{|c|}{ SFR\% } & \multicolumn{2}{c|}{ VVP\% } & \multirow{2}{*}{ CI\% } \\
\cline { 2 - 5 } & Det & CC & Det & CC & \\
\hline 0.10 & 60.01 & 8.98 & 4.67 & 0.14 & 2.98 \\
\hline 0.15 & 63.95 & 4.87 & 6.36 & 0.18 & 4.60 \\
\hline 0.20 & 67.10 & 6.21 & 7.65 & 0.35 & 4.35 \\
\hline 0.25 & 74.82 & 3.54 & 10.06 & 0.27 & 5.88 \\
\hline
\end{tabular}

TABLE VI

SECURITy CONSTRAINT VIOLATIONS COMPARISON FOR THE 190 BUS SYSTEM

\begin{tabular}{|c|c|c|c|c|c|}
\hline \multirow{2}{*}{$\sigma^{d}$} & \multicolumn{2}{|c|}{ SFR\% } & \multicolumn{2}{c|}{ VVP\% } & \multirow{2}{*}{ CI\% } \\
\cline { 2 - 5 } & Det & CC & Det & CC & \\
\hline 0.10 & 65.82 & 5.49 & 8.27 & 0.59 & 4.42 \\
\hline 0.15 & 71.00 & 6.83 & 11.18 & 0.57 & 4.50 \\
\hline 0.20 & 75.25 & 4.53 & 14.05 & 0.90 & 3.89 \\
\hline 0.25 & 77.57 & 6.47 & 16.65 & 0.18 & 6.38 \\
\hline
\end{tabular}

factor known as conservative index (CI), which is defined by:

$$
C I=\frac{P l_{C C}-P l_{D C}}{P l_{D C}}
$$

where $P l_{C C}$ denotes expected value of base case power loss with the controlling devices fixed at the schedule obtained from STS-VVC program, and $P l_{D C}$ represents the expected value of base case power loss with the controlling devices fixed at the schedule obtained from deterministic VVC program. Thus the conservative index can be defined as the increase in the expected power loss in the system for the chance constrained schdule from that of the base schedule. The lower the values of this metric, the better the algorithm is performing. In order to calculate the expectations of the system power loss, probabilities are assigned to each scenario by the method discussed in [12].

The words 'Det' and ' $\mathrm{CC}$ ' used in various tables represent deterministic case and chance constrained case respectively. Tables II and III display the comparison of solutions of deterministic VVC and STS-VVC approaches from the perspective of the values attained by the reactive power controlling devices for the UKGDS 95 bus system; with the resulting voltage violations and the corresponding conservativeness indices shown in Table IV. In order to keep the presentation simple, the corresponding tables for control device set points have been omitted for the remaining two test systems. However, Tables V and VI present the robustness and conservativeness parameters for these systems. Tables IV-VI clearly show that the performance of the base case schedule deteriorates significantly with the increasing deviation in the DG forecast error. This is evident because the forecast errors become more prominent with the increasing standard deviation of the probability distribution.

It is evident from these tables that the voltage violations present in the base case schedule are effectively removed by the STS-VVC schedule. With the base case schedules, more than $40 \%$ of the scenarios suffer from security constraint violations on at least one node in every case. The corresponding schedules for the STS-VVC version are more robust and are able to eliminate the voltage violations in almost all
TABLE VII

COMPUTATIONAL PERformance AND Relaxation Gap Comparison FOR THE 95 BUS UKGDS SYSTEM

\begin{tabular}{|c|c|c|c|c|c|}
\hline & $\sigma^{d}$ & $\begin{array}{c}\text { Max. SOC } \\
\text { Relax. Gap }\end{array}$ & $\begin{array}{c}\text { CPU } \\
\text { time(s) }\end{array}$ & $\begin{array}{c}\text { \# of } \\
\text { iter. }\end{array}$ & $\begin{array}{c}\text { Scenarios } \\
\text { considered }\end{array}$ \\
\hline \multirow{2}{*}{ Det } & - & $3.2 \times 10^{-8}$ & 2.61 & - & - \\
\hline \multirow{4}{*}{ CC } & 0.10 & $2.0 \times 10^{-7}$ & 48.12 & 2 & 6 \\
\cline { 2 - 6 } & 0.15 & $1.7 \times 10^{-8}$ & 97.34 & 3 & 11 \\
\cline { 2 - 6 } & 0.20 & $2.1 \times 10^{-8}$ & 109.54 & 3 & 13 \\
\cline { 2 - 6 } & 0.25 & $1.3 \times 10^{-7}$ & 102.4 & 3 & 12 \\
\hline
\end{tabular}

TABLE VIII

COMPUTATIONAL PERFormanCE AND RELAXATION GAP COMPARISON FOR THE IEEE 123 BUS SYSTEM

\begin{tabular}{|c|c|c|c|c|c|}
\hline & $\sigma^{d}$ & $\begin{array}{c}\text { Max. SOC } \\
\text { Relax. Gap }\end{array}$ & $\begin{array}{c}\text { CPU } \\
\text { time(s) }\end{array}$ & $\begin{array}{c}\text { \# of } \\
\text { iter. }\end{array}$ & $\begin{array}{c}\text { Scenarios } \\
\text { considered }\end{array}$ \\
\hline Det & - & $6.1 \times 10^{-8}$ & 4.32 & - & - \\
\hline \multirow{4}{*}{ CC } & 0.10 & $1.0 \times 10^{-7}$ & 134.62 & 3 & 14 \\
\cline { 2 - 6 } & 0.15 & $8.2 \times 10^{-7}$ & 130.21 & 3 & 12 \\
\cline { 2 - 6 } & 0.20 & $3.4 \times 10^{-7}$ & 136.72 & 3 & 13 \\
\cline { 2 - 6 } & 0.25 & $1.5 \times 10^{-7}$ & 210.63 & 4 & 18 \\
\hline
\end{tabular}

TABLE IX

Computational Performance AND Relaxation Gap Comparison FOR THE 190 BUS SYSTEM

\begin{tabular}{|c|c|c|c|c|c|}
\hline & $\sigma^{d}$ & $\begin{array}{c}\text { Max. SOC } \\
\text { Relax. Gap }\end{array}$ & $\begin{array}{c}\text { CPU } \\
\text { time(s) }\end{array}$ & $\begin{array}{c}\text { \# of } \\
\text { iter. }\end{array}$ & $\begin{array}{c}\text { Scenarios } \\
\text { considered }\end{array}$ \\
\hline \multirow{3}{*}{ Det } & - & $1.5 \times 10^{-8}$ & 6.08 & - & - \\
\hline \multirow{4}{*}{ CC } & 0.10 & $9.9 \times 10^{-8}$ & 123.12 & 3 & 12 \\
\cline { 2 - 6 } & 0.15 & $2.5 \times 10^{-7}$ & 119.34 & 3 & 10 \\
\cline { 2 - 6 } & 0.20 & $1.7 \times 10^{-7}$ & 191.71 & 4 & 17 \\
\cline { 2 - 6 } & 0.25 & $2.3 \times 10^{-7}$ & 268.09 & 4 & 20 \\
\hline
\end{tabular}

the scenarios. It can also be noted from these tables that the highest value attained by VVP in any case corresponding to the STS-VVC schedule is $0.90 \%$, hence conforming with a priori probability bounds set for in (14). The price of robustness of the chance constrained solutions is a slight increase in power loss of the system. This slight conservativeness can be tradedoff for the robustness of the solutions because robustness is a desirable feature in power system operation where the infeasible operation results in high penalty.

Fig. 4 shows the boxplot of the voltage for the most stressed bus (MSB) across all the MC aposteriori trials when the $\sigma^{d}$ is set at 0.20 in the UKGDS 95 bus system. The MSB for the base case schedule turns out to be the bus 95 which has a voltage swell close to 1.09 in the extreme situation. Even the median of the box plot is very close to the upper statutory limit of 1.05 and the third quartile of the box plot overshoots this limit. On the contrary, it is noticed that the voltages for all the scenarios for the MSB in the STS-VVC are within the statutory limits. Note that the MSB is determined by the maximum value of the boxplot and not the outliers. This explains why there is a small scenario failure rate of $0.11 \%$ corresponding to this case as shown in Table IV, as the outliers of some other nodes are shooting past the upper limit of 1.05 .

\section{Comparison of Computational Performance Between Two Stage Stochastic and Deterministic VVC Approaches}

The computational performance of the base case and STSVVC schemes is compared in the Tables VII-IX on the basis of 


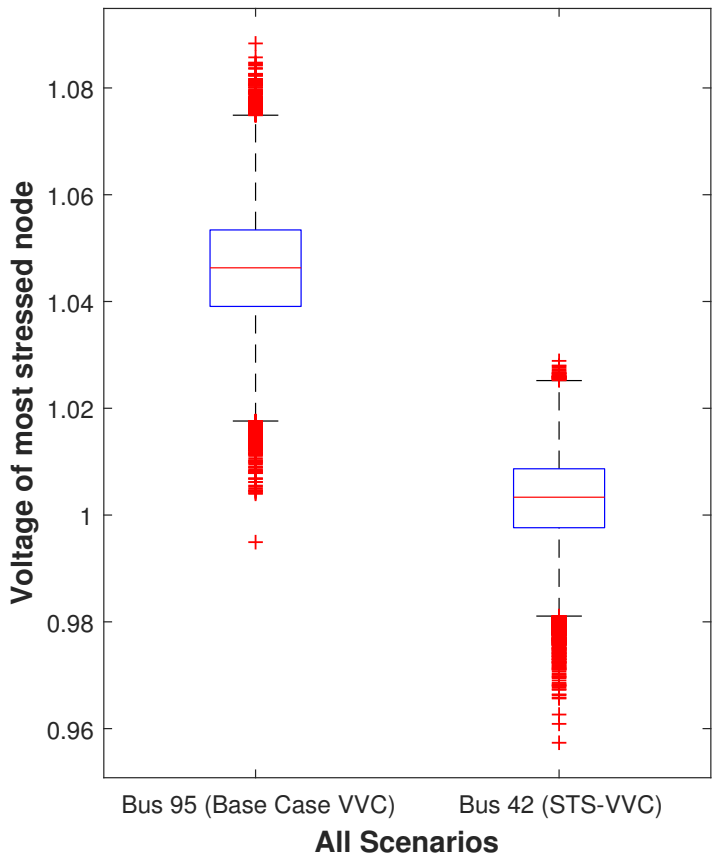

Fig. 4. Comparison of the most severely stressed bus due to the base case and STS-VVC schedules for the UKGDS 95 bus system

TABLE X

EFFECT OF $C_{s}$ ON TAP COUNT MINIMIZATION AND POWER LOSS FOR THE 190 BUS SYSTEM.

\begin{tabular}{|c|c|c|c|}
\hline$C_{s}$ & $\begin{array}{c}\text { Number of } \\
\text { Cap Switching }\end{array}$ & $\begin{array}{c}\text { Number of } \\
\text { OLTC Switching }\end{array}$ & $\begin{array}{c}\text { Normalized } \\
\text { power loss }\end{array}$ \\
\hline 0 & 12 & 12 & 1 \\
\hline 0.25 & 12 & 6 & 1.043 \\
\hline 0.50 & 6 & 7 & 1.073 \\
\hline 0.75 & 4 & 5 & 1.103 \\
\hline 1 & 2 & 5 & 1.121 \\
\hline
\end{tabular}

total CPU time and the number of iterations required to reach to the final optimum schedule. The number of iterations taken by the STS-VVC algorithm is small, which implies that the algorithm needs to update its schedule only a couple of times before it can find a robustly feasible optimum point. However, the time taken by the STS-VVC algorithm is significantly large as compared to the deterministic case. This is because STSVVC needs to check for violated scenarios by running load flows for each of the generated scenarios along with running a scenario reduction routine after each iteration, while the deterministic case does not make use of any of these routines.

Finally, results from these tables also illustrate that the conic relaxation gap for any case is less than $10^{-7}$, which means that the conic relaxation is exact.

\section{Effect of tap minimization}

The results in Table X have been obtained for the 190 bus system with a standard deviation of 0.15 in the DG forecast errors. The old schedule for all the transformers is taken as 1.0 and for the SSCs it is taken to be $240 \mathrm{kvar}$. It can be seen as the value of $C_{s}$ increases towards 1 , the number of switching operations from the previous schedule decreases which also results in increase in the power loss of the system. The power loss of the system has been normalized against the particular case when only power loss minimization is considered in the objective i.e. when $C_{s}=0$.

\section{CONCLUSION}

This paper presents a two-stage stochastic chance constrained VVC approach to achieve robust solutions in the face of uncertainty in DG active power and consumer loads. Convexification and conic relaxation of power flow equations along with replacing probabilistic chance constraints with enough scenarios from the corresponding probability spaces enables to cast the problem as an MISOCP. Initially the proposed solution algorithm only considers the deterministic case but enforces the most dominant violated scenarios in subsequent iterations until it reaches its robust optimum point. The algorithm is not only able to dispatch a schedule for classical reactive power controlling devices but also optimizes quadratic decision rules relating reactive power of DGs with their real power injection. The numerical results reveal that the proposed algorithm has better performance as compared to the traditional deterministic case and is able to achieve highly robust solutions. The price of robustness is paid in terms of slight conservativeness in the solutions.

\section{ACKNOWLEDGEMENT}

The authors would like to thank Department of Electrical \& Electronic Engineering, Imperial College London and Inlaks Shivdasani Foundation for funding the research. This work is also partly funded through JUICE project (contract no: $\mathrm{EP} / \mathrm{P} 003605 / 1)$.

The work of R. A. Jabr on this project has been funded with support from the National Council for Scientific Research in Lebanon and the American University of Beirut, for which the authors are highly grateful.

\section{REFERENCES}

[1] I. Roytelman, B. K. Wee, R. L. Lugtu, T. M. Kulas, and T. Brossar, "Pilot project to estimate the centralized volt/VAr control effectiveness,"IEEE Trans. Power Syst., vol. 13, no. 3, pp. 864-869, Aug. 1998.

[2] J. J. Grainger and S. Civanlar, " Volt/Var Control on Distribution Systems with Lateral Branchces Using Shunt Capacitors and Voltage Regulators: parts IIII," IEEE Trans. Power App. and Syst., vol. 104, no. 11, pp. 32783297, 1985.

[3] T. Senjyu, Y. Miyazato, A. Yona, N. Urasaki and T. Funabashi, "Optimal Distribution Voltage Control and Coordination With Distributed Generation," IEEE Trans. Power Del., vol. 23, no. 2, pp. 1236-1242, April 2008.

[4] M. B. Liu, C. A. Canizares, and W. Huang, "Reactive Power and Voltage Control in Distribution Systems With Limited Switching Operations," IEEE Trans. Power Syst., vol. 24, no. 2, pp. 889-899, May 2009.

[5] A. Borghetti, "Using mixed integer programming for the volt/VAr optimization in distribution feeders," Elect. Power Syst. Research, vol. 98, pp. 39-50, May 2013.

[6] F. C. Lu and Y. Hsu, "Reactive power/voltage control in a distribution system using dynamic programming," IEE proceedings Generation, Transmission and Distribution, vol. 142, no. 6, pp. 639-645, Nov. 1995.

[7] R. H. Liang and C. K. Cheng, "Dispatch of main transformer ULTC and capacitors in a distribution system," IEEE Trans. Power Del., vol. 16, no. 4, pp. 625-630, Oct. 2001.

[8] A. Augugliaro, L. Dusonchet, S. Favuzza, and E.R. Sanseverino, "Voltage regulation and power losses minimization in automated distribution networks by an evolutionary mutiobjective approach," IEEE Trans. Power Syst., vol. 19, no. 3, pp. 1516-1527, Aug. 2004. 
[9] S. Auchariyamet and S. Sirisumrannukul, "Optimal daily coordination of volt/VAr control devices in distribution systems with distributed generators," 45th Intern. Universities Power Eng. Conference UPEC2010, Cardiff, Wales, pp. 1-6, 2010.

[10] A. Collinson, F. Dai, A. Beddoes, and J. Crabtree, "Solutions for the connection and operation of distributed generation," EA Technology Ltd, Technical Report, Jul. 2003.

[11] L. Miller, L. Cibulka, M. Brown, A. V. Meier, and R. Arghandeh, "Distribution system voltage management and optimization for integration of renewables and electric vehicles: status and state of the art," California institute for energy and environment, Report, 2013.

[12] T. Niknam, M. Zare, and J. Aghaei, "Scenario-based multiobjective volt/var control in distribution networks including renewable energy sources," IEEE Trans. Power Del., vol. 27, no. 4, pp. 2004-2019, Oct. 2012.

[13] T. Ding, S. Liu, W. Yuan, Z. Bie, and B. Zeng, "A two-stage robust reactive power optimization considering uncertain wind power integration in active distribution networks," IEEE Trans. Sust. Energy, vol. 7, no. 1, pp. 301-311, Jan. 2016.

[14] Y. P. Agalgaonkar, B. C. Pal, and R. A. Jabr, "Stochastic distribution system operation considering voltage regulation risks in the presence of PV generation," IEEE Trans. Sust. Energy, vol. 6, no. 4, pp. 1315-1324, Oct. 2015.

[15] Y. Wang, Y. Liu, and D. S. Kirschen, "Scenario reduction with submodular optimization," IEEE Trans. Power Syst., vol. 32, no. 3, pp. 2479-2480, May 2017.

[16] M. Farivar and S. H. Low, " Branch flow model: Relaxations and convexifications - part I and II," IEEE Trans. Power Syst., vol. 28, no. 3, pp. 2554 - 2572, Aug. 2013.

[17] "IEEE standard for interconnecting distributed resources with electric power systems," IEEE Std 1547-2003, pp. 1-28, July 2003.

[18] "IEEE standard for interconnecting distributed resources with electric power systems - amendment 1," IEEE Std 1547a-20014 (Amendment to IEEE Std 1547-2003), pp. 1-16, May 2014.

[19] H. Zhang and P. Li, "Chance constrained programming for optimal power flow under uncertainty," IEEE Trans. Power Syst., vol. 26, no. 4, pp. 2417 - 2424, Nov. 2011.

[20] S. K. Ibrahim, A. M. Cramer, and Y. Liao, "Integrated distributed system optimization using a chance-constrained formulation," 2017 North Amer. Power Symp., Morgantown, USA, 17-19 Sept. 2017.

[21] H. Ahmadi, J. R. Martì, and H. W. Dommel, "A Framework for VoltVAR Optimization in Distribution Systems," IEEE Trans. Smart Grid, vol. 6, no. 3, pp. 1473-1483, May 2015.

[22] P. M. Esfahani, T. Sutter, and J. Lygeros, "Performance Bounds for the Scenario Approach and an Extension to a class of Non-Convex Programs," IEEE Trans. Aut. Control, vol. 60, no. 1, pp 46-58, Jan. 2015.

[23] R. A. Jabr, N. Martins, B. C. Pal, and S. Karaki, "Contingency constrained VAr planning using penalty successive conic programming," IEEE Trans. Power syst., vol. 27, no. 1, pp. 545-553, Feb. 2012.

[24] A. Samadi, R. Eriksson, L. Söder, B. G. Rawn, and J. C. Boemer, "Coordinated active power-dependent voltage regulation in distribution grids with PV systems," IEEE Trans. Power Del., vol. 29, no. 3, pp. 14541464, Jun. 2014.

[25] R. A. Jabr, "Linear decision rules for control of reactive power by distributed photovoltaic generators," IEEE Trans. Power Syst., vol. 33, no. 2, pp. 2165-2174, Mar. 2018.

[26] D. K. Molzahn, B. C. Lesieutre, and C. L. DeMarco, "Approximate representation of ZIP loads in a semidefinite relaxation of the OPF problem," IEEE Trans. Power Syst., vol. 29, no. 4, pp. 1864-1865, Jul. 2014.

[27] J. Aghaei, T. Niknam, R. Azizipanah-Abarghooee, and J. M. Arroyo, "Scenario-based dynamic economic dispatch considering load and wind power uncertainties," Int. J. Elect. Power Energy Syst., vol. 47, pp. 351367, 2013.

[28] I. Džafić, R. A. Jabr, E. Halilovic, and B. C. Pal, “A sensitivity approach to model local voltage controllers in distribution networks," IEEE Trans. Power Syst., vol. 29, no. 3, pp. 1419-1428, May 2014.

[29] Y. Wang, Y. Liu, and D. S. Kirschen, "Scenario reduction with submodular optimization,” IEEE Trans. Power Syst., vol. 32, no. 3, pp. 2479-2480, May 2017.

[30] N. Gröwe-Kuska, H. Heitsch, and W. Römisch, "Scenario reduction and scenario tree construction for power management problems," in Proc. IEEE Bologna PowerTech Cong., 2003, pp. 1-7.

[31] Y. P. Agalgaonkar, B. C. Pal, and R. A. Jabr, "Distribution voltage control considering the impact of PV generation on tap changers and autonomous regulators," IEEE Trans. Power Syst., vol. 29, no. 1, pp. $182-$ 192, Jan. 2014.
[32] R. A. Jabr, "Adjustable robust OPF with renewable energy sources," IEEE Trans. Power Syst., vol. 28, no. 4, pp. 4742-4751, Nov. 2013.

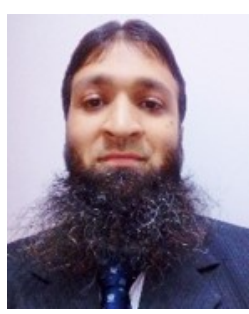

Firdous Ul Nazir received his B.Tech degree in electrical engineering from NIT Srinagar, India, in 2012, and his M.Tech degree in electrical power systems from IIT Roorkee, India, in 2015. He is currently working towards his Ph.D. degree at the Department of Electrical and Electronic Engineering, Imperial College London, London, U.K. His current research interests include distribution system modelling, operation and control, and optimization theory.

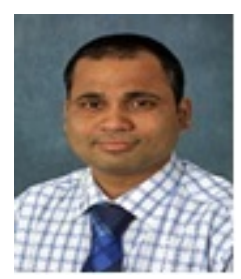

Bikash C. Pal (M'00-SM'02-F'13) recieved the B.E.E. (with honors) degree from Jadavpur University, Calcutta, India, M.E. degree from the Indian Institute of Science, Bangalore, India, and Ph.D. degree from Imperial College London, London, U.K, in 1990, 1992, and 1999, respectively, all in electrical engineering.

Currently, he is a Professor with the Department of Electrical and Electronic Engineering, Imperial College London. He was Editor-in-Chief of IEEE Transactions on Sustainable Energy (2012-2017) and Editor-in-Chief of IET Generation, Transmission and Distribution (2005-2012) and is Fellow of IEEE for his contribution to power system stability and control. His current research interests include renewable energy modelling and control, state estimation, and power system dynamics.

Rabih A. Jabr (M'02-SM'09-F'16) was born in Lebanon. He received the B.E. degree in electrical engineering (with high distinction) from the American University of Beirut, Beirut, Lebanon, in 1997 and the Ph.D. degree in electrical engineering from Imperial College London, U.K., in 2000. Currently, he is a Professor in the Department of Electrical and Computer Engineering at the American University of Beirut. His research interests are in mathematical optimization techniques and power system analysis and computing. 